\title{
Calidad de semilla de moringa y su adaptabilidad en campo en asociación con zacate buffel
}

\section{Seed quality of moringa and its adaptability in the field in association with buffelgrass}

Martha Gómez-Martínez 1 (1)

Raúl Rodríguez-Herrera (D),

Jorge R. González-Domínguez 2 (D)

Marisol Santos-Fernández 2 [D]

Susana Gómez-Martínez 2 [D

${ }^{1}$ Facultad de Ciencias Químicas, Universidad Autónoma de Coahuila.

Blvd. Venustiano Carranza e Ing. José Cárdenas V. s/n, CP. 25280. Saltillo, Coahuila, México.

${ }^{2}$ Universidad Autónoma Agraria Antonio Narro. Calzada Antonio Narro, 1923. CP. 25315. Buenavista Saltillo, Coahuila, México.

*Autor de correspondencia:

sgomart@gmail.com

Artículo científico

Recibido: 30 de agosto de 2019

Aceptado: 01 de mayo de 2020

Como citar: Gómez-Martínez M Rodríguez-Herrera R, GonzálezDomínguez JR, Santos-Fernández M, Gómez-Martínez S (2020) Calidad de semilla de moringa y su adaptabilidad en campo en asociación con zacate buffel. Ecosistemas y Recursos Agropecuarios 7(2): e2408. DOI: 10.19136/era.a7n2.2408

RESUMEN. Moringa oleifera presenta adaptación a zonas tropicales y semiáridas, y tiene hojas con alto contenido de proteína. En dos tipos de moringa (cápsula corta y cápsula larga) se evalúo el porcentaje de germinación, emergencia, altura de planta, velocidad de emergencia, y crecimiento en condiciones de vivero. La adaptación en campo se evaluó en una mezcla de semilla de ambos tipos. La cápsula larga presentó un 80 y 70\% de germinación y emergencia, respectivamente. La velocidad de emergencia fue similar en ambos tipos de moringa, mientras que el crecimiento en cápsula larga fue de 2.02 y en corta de $1.24 \mathrm{~mm} \mathrm{día}^{-1}$. La incidencia de hongos fitopatógenos (Fusarium oxysporum) en semilla con testa, sin testa y solo testa, fue mayor en cápsula corta en $70 \%$. Las plantas fueron atacadas por nematodos Dorylaimus que se encontraron en el sustrato. En campo se tuvo $97 \%$ de establecimiento, $31 \%$ de la población fue destruida por hormigas arrieras, competencia del zacate buffel y bajas temperaturas. La mayor calidad fisiológica de semilla se observó en el tipo cápsula larga, con mayor porcentaje de germinación e índice de velocidad de crecimiento que la cápsula corta, y menor incidencia de Fusarium oxysporum. Los resultados indican que $M$. oleifera tiene potencial de adaptación en la región semiárida del norte de México.

Palabras clave: Competencia interespecífica, fitotoxicidad, Moringa oleifera, nematodos, vigor de semilla.

ABSTRACT. Moringa oleifera presents adaptation to tropical and semi-arid zones, and has leaves with high protein content. In two types of moringa (short capsule and long capsule), the percentage of germination, emergence, plant height, speed of emergence and growth under nursery conditions were evaluated. The field adaptation was a mixture of seed of both types. The long capsule showed 80 and $70 \%$ of germination and emergence, respectively. The emergence speed was similar in both types of moringa, while the growth rate in long capsule was 2.02 and in short capsule $1.24 \mathrm{~mm}_{\text {day }}{ }^{-1}$. The incidence of phytopathogenic fungi (Fusarium oxysporum) in seed with testa, seed without testa and only the testa, was higher in short capsule type with $70 \%$. The plants were attacked by Dorylaimus nematodes, which were found in the substrate. In field conditions, there was $97.37 \%$ of plant establishment, but, $31 \%$ of the population was destroyed by leaf cutter ants, buffel grass competition and low temperatures. The highest physiological seed quality was obtained by the long capsule type, since, it had a germination percentage and a speed of growth higher than the short capsule, and lower incidence of Fusarium oxysporum. These results indicate that $M$. oleifera has potential for adaptation in the semiarid region of northern Mexico.

Key words: Interspecific competition, phytotoxicity, Moringa oleifera, nematodes, seed vigor. 


\section{INTRODUCCIÓN}

México es uno de los países más vulnerables ante el cambio climático, por sus limitaciones técnicas, pobreza y condición de país en desarrollo (Hernández et al. 2018). Las actividades agrícolas ocasionan emisiones de gases de efecto invernadero, pero también contribuyen en forma importante a minimizar estos efectos, mediante la reducción de la vulnerabilidad de los cultivos. De acuerdo al reporte de la Cuarta Evaluación del IPCC sobre el cambio climático, el sector agrícola será el más afectado (Easterling et al. 2007). Del total de las emisiones de gases de efecto invernadero a nivel mundial, el 12.3\% provienen de la agricultura mexicana, de las cuales el $50.4 \%$ proceden del sector agrícola y el $49.4 \%$ del agropecuario (Gutiérrez et al. 2013).

La región árida y semiárida en México es poco más del $50 \%$ del territorio nacional con más de 100 millones de hectáreas, donde la actividad económica más importante es la ganadería extensiva (Gómez et al. 2007). Al respecto Magaña y Neri (2012) reportan que en los próximos años se tendrán sequías más extensas por lo que se requiere estudiar especies de plantas forrajeras aptas para producir bajo ambientes de estrés de calor y sequía, para responder al cambio climático (Ruiz 2012). Por lo que el desarrollo de sistemas silvopastoriles de producción forrajera puede contribuir a reducir la vulnerabilidad del semidesierto al cambio climático, siendo la moringa (Moringa oleifera) y el zacate buffel (Pennisetum ciliare L.) dos especies candidatas por la condición arbórea de la primera y herbácea de la segunda (Conant et al. 2005). Tanto la moringa como el zacate buffel son de rápido crecimiento en el verano, perennes, tolerantes a la sequía y al calor, pero pueden morir con temperaturas de $3 \mathrm{a}-1{ }^{\circ} \mathrm{C}$ y de -5 a $-7{ }^{\circ} \mathrm{C}$, respectivamente (Olson y Fahey 2011, Padilla et al. 2017). En México, M. oleifera se distribuye a lo largo de toda la costa del Pacífico (Olson y Alvarado 2016) y en el noreste de México tiene potencial de cultivo (Meza-Carranco et al. 2016). Es una alternativa forrajera y alimentaria (Pérez 2010), y resiste hasta seis meses la sequía (Mendieta-Araica et al. 2013). Además sus hojas, flores, frutos y raíces, se utilizan como alimento humano (Toral et al. 2013, Gómez-Martínez et al. 2020). En los últimos 10 años ha tenido un gran auge por sus propiedades nutritivas, farmacocinéticas e industriales (Lakshmipriya et al. 2016, Tesfay et al. 2016), lo que puede contribuir a elevar el nivel de vida de las comunidades rurales de las zonas áridas (Hernández et al. 2018).

En lo referente al trasplante, es una técnica que asegura alto porcentaje de establecimiento, ya que hay un mayor control de la maleza, por lo que es necesario el uso de semilla de buena calidad fisiológica que asegure la producción de plántulas sanas y vigorosas (Oswald y Ransom 2002). Pero patógenos como nematodos y hongos pueden afectar en estado de plántulas, causando amarillamiento, marchitamiento, caída de hoja o pudrición del tallo (Espinoza-Ahumada et al. 2019). Mientras que los hongos son los principales causantes del deterioro de la semilla, debido al periodo de almacenamiento y falta de condiciones adecuadas, lo que causa disminución de la germinación y emergencia de la plántula (Krishnamurthy et al. 2008). Debido a lo anterior, el objetivo del presente estudio fue evaluar la calidad fisiológica, el vigor de dos tipos de moringa e identificar los patógenos que causan daño a las plántulas en vivero, además de conocer su adaptabilidad a las condiciones climáticas del norte de México en asociación con zacate buffel.

\section{MATERIALES Y MÉTODOS}

La investigación se realizó en el vivero y el laboratorio de Fitopatología de la Universidad Autónoma Agraria Antonio Narro (UAAAN) en Saltillo, Coahuila. Mientras que la evaluación de adaptabilidad en el Campo Experimental de Zaragoza, ubicado en el municipio de Zaragoza, Coahuila. El clima de la región es seco en verano y frío en invierno extremoso, temperatura promedio de 22 a $24^{\circ} \mathrm{C}$, y precipitación promedio anual de 300 a 400 mm (CONAGUA 2015).

\section{Material vegetal}

Se utilizó semilla de dos tipos de $M$. oleifera que se denominaron cápsula corta $(15$ a $25 \mathrm{~cm}$ ) y cápsula larga $(30$ a $80 \mathrm{~cm}$ ) por el tamaño de fruto 
(Castillo-López et al. 2017). La semilla fue proporcionada por la Facultad de Agronomía de la Universidad Autónoma de Nuevo León y se mantuvo almacenada por 18 meses a temperatura ambiente. En campo se estableció una mezcla de plantas de cápsula corta y larga, y el híbrido AN17PS de zacate buffel de la UAAAN.

\section{Porcentaje de germinación}

Se determinó en el laboratorio en 100 semillas de moringa cápsula corta y larga, las cuales se desinfectaron con hipoclorito de sodio al $1 \%$ por un minuto y enjuagaron con agua en un vaso de precipitados de $500 \mathrm{~mL}$ por $2 \mathrm{~min}$. La siembra se realizó en cajas Petri con papel filtro humedecido como substrato, además de solución fungicida de captan al 1\% para prevenir la contaminación por hongos. Las cajas Petri se colocaron en una germinadora Seedburo ${ }^{\circledR}$ a temperatura de $25{ }^{\circ} \mathrm{C} \pm 1$. La germinación se registró todos los días a partir del octavo día de siembra hasta el día 21. Se tomó como semilla germinada aquella que tenía una radícula de $2 \mathrm{~cm}$ de longitud y como semilla anormal aquella que no alcanzó los $2 \mathrm{~cm}$.

\section{Evaluación en vivero}

La siembra en invernadero se realizó en charolas de poliuretano de 60 cavidades con peat moss como substrato. Se sembraron 60 semillas por repetición de cada tipo, lavadas y desinfectadas previamente. El experimento se estableció bajo un diseño completamente al azar con tres repeticiones. En vivero se determinó el porcentaje de emergencia, que se registró durante 21 días a partir del día 16 de la siembra. Mientras que la altura de planta se midió a los 37, 44 y 51 días después de la siembra, tomando la altura desde la base hasta el ápice de la planta en una muestra de 10 plantas de cada tipo de moringa por repetición. El índice de velocidad de emergencia (número de plantas día ${ }^{-1}$ ) se cuantificó todos los días y se consideró como día inicial cuando apareció la primera plántula hasta los 21 días después. El vigor se calculó con la fórmula propuesta por Maguire (1962):

$$
I V E=\sum \frac{N P}{D}+\frac{N P}{D}+\frac{N P}{D}+\ldots \frac{N P}{D}
$$

Donde: IVE = Índice de velocidad de emergencia, NP = número de plántulas emergidas, y $\mathrm{D}$ = días después de la siembra.

La velocidad de crecimiento ( $\mathrm{mm}$ día ${ }^{-1}$ ) se estimó de manera indirecta con las diferencias de altura de planta entre el tiempo transcurrido entre las dos lecturas:

$$
V C=\frac{\left(A_{2}-A_{1}\right)}{T_{2}-T_{1}}
$$

Donde: $\mathrm{VC}=$ Velocidad de crecimiento, $\mathrm{A}=$ altura, $\mathrm{y}$ $\mathrm{T}=$ tiempo.

Los datos se analizaron por la prueba de TStudent $(\alpha=0.05)$. La altura de planta, índice de velocidad de emergencia e índice de velocidad de crecimiento se determinaron a los 21 días después de inicio de la emergencia, debido a que las plantas después de la tercera semana de emergencia presentaron signos de pudrición en tallo y raíz.

\section{Identificación de nematodos y hongos fi- topatógenos}

Se analizaron las semillas de los dos tipos de moringa y el peat moss que se utilizó como substrato, para identificar los patógenos que pudieran estar asociados con la pérdida de vigor y muerte de las plantas en vivero. La observación de los nematodos se determinó en $7 \mathrm{~g}$ de peat moss que se colocó en una malla metálica $20 \times 20 \mathrm{~cm}$ y kleenex en la parte superior de un embudo de Baermann para luego verter $200 \mathrm{~mL}$ de agua corriente y dejar reposar $48 \mathrm{~h}$. Posteriormente, se filtró en un tubo de ensayo y se tomó una muestra de la que se colocaron tres gotas en un vidrio de reloj para observar en un estereomicroscopio. Posteriormente, se montaron en portaobjetos con ayuda de una aguja de disección agregando una gota de lactofenol. La muestra se cubrió con un cubreobjeto y se selló con esmalte, para luego observar al microscopio. La identificación de los nematodos a nivel de género se realizó con base en las características morfológicas (Cepeda 1996).

El aislamiento de los hongos se realizó en una muestra de 50 semillas de los dos tipos de moringa, las cuales se dividieron en dos grupos: 25 semillas se sembraron sin eliminar testa y alas, y de las otras 
25 semillas se sembró el embrión y la testa por separado, teniendo la siembra de semilla con testa, semilla sin testa y solo la testa de cada tipo de moringa. Las semillas se desinfectaron con hipoclorito de sodio al $3 \%$ por un minuto, se enjuagaron con agua estéril tres veces por tres minutos y se secaron con papel de estraza estéril. Para la siembra, se utilizó el medio de cultivo de agar-dextrosa-papa (PDA) y cámara húmeda (papel filtro), para favorecer el crecimiento de hongos. Las observaciones de crecimiento bacteriano se realizaron a las 24 y 48 y el crecimiento de hongos a las $72 \mathrm{~h}$. A los 7 días se tomaron explantes con un sacabocados de cada crecimiento fúngico para realizar resiembras en PDA, para identificar las especies de hongos, los cultivos se dejaron esporular por 15 días y se identificaron con el manual del CIMMyT (Warham et al. 1997).

\section{Siembra en campo}

En un vivero de la UAAAN, se sembró en charolas de poliuretano de 60 cavidades una mezcla de semilla de moringa cápsula corta y larga, y en charolas de 200 cavidades la semilla del híbrido de zacate buffel, en la siembra de moringa y zacate se utilizó como medio de crecimiento peat moss. La siembra se realizó depositando una semilla por cavidad, el riego se realizó todos los días, y la fertilización cada semana con la fórmula 9-45-15 N-P-K, vía riego. A los 70 días después de la siembra las charolas con las plantas de moringa se llevaron al campo experimental en Zaragoza, para su trasplante, previo a este se realizó barbecho, rastreo y bordeo. El experimento se estableció en un suelo arcilloso ( $50 \%$ de arcilla), medianamente alcalino $(\mathrm{pH}$ 8.3) y no salino (0.05 mmhos $\mathrm{cm}^{-1}$ ). Después del trasplante se aplicó un riego de una lámina de $10 \mathrm{~cm}$. Una semana después, se realizó el trasplante del zacate buffel de forma manual, a distancia de $0.50 \mathrm{~m}$ entre plantas y de 0.80 $\mathrm{m}$ entre surcos, para luego realizar un segundo riego.

Los tratamientos evaluados fueron las densidades de población de moringa de 15, 40 y 140 árboles por parcela de $120 \mathrm{~m}^{2}$. Para la población de 15 árboles por parcela se utilizaron tres surcos, se trasplantaron cinco árboles por surco a distancia de 4 $\mathrm{m}$ entre árboles y $2.40 \mathrm{~m}$ entre surcos, para tener una densidad de 1250 árboles ha $^{-1}$. En la población de 40 árboles por parcela se usaron cuatro surcos, en los que se trasplantaron 10 árboles por surco a distancia de $2 \mathrm{~m}$ entre árboles y $1.60 \mathrm{~m}$ entre surcos, para una densidad de 3300 plantas ha $^{-1}$. Para la población de 140 árboles por parcela se utilizaron siete surcos, en los que se trasplantaron 20 árboles por surco a distancia de $1 \mathrm{~m}$ entre árbol y $0.80 \mathrm{~m}$ entre surcos para una densidad de 11660 plantas ha $^{-1}$. Mientras que el trasplante del zacate buffel solo se realizó en los bloques I y II en los surcos libres y entre los árboles de moringa. En tanto que en el bloque III no se trasplantó zacate buffel con el propósito de observar el comportamiento de los árboles de la moringa sin competencia.

En campo se determinó el porcentaje de establecimiento un mes después del trasplante, por medio del conteo del número de árboles y plantas de zacate buffel vivos, para luego transformar a porcentajes. El efecto de la competencia entre especies se determinó con el número de árboles de moringa y plantas de zacate buffel vivos por unidad experimental. La altura de los árboles de moringa y de zacate buffel se midió en los meses de septiembre de 2014 y marzo de 2015, en moringa la altura se tomó desde la base hasta el ápice del tallo principal del árbol. Mientras que en zacate buffel se tomó desde la base hasta la mayoría de las panículas. En la medición del mes de marzo se contó el número de árboles que fructificaron y el número de frutos por árbol. La evaluación se realizó bajo un diseño de bloques completos al azar con tres tratamientos y tres repeticiones, el tamaño de las unidades experimentales fue de $120 \mathrm{~m}^{2}$.

\section{Diseño experimental}

Las variables de respuesta de los experimentos de laboratorio e invernadero: porcentaje de germinación, porcentaje de emergencia, IVE, velocidad de crecimiento y altura de planta se analizaron mediante la prueba de T-Student $(\alpha=0.05)$. Mientras que el porcentaje de establecimiento, porcentaje de sobrevivencia, número de árboles con frutos y frutos por árbol, se realizaron bajo análisis de varianza y comparación de medias por medio de la prueba de 
diferencia mínima significativa $(\alpha<0.05)$.

\section{RESULTADOS}

\section{Evaluación en laboratorio y vivero}

La germinación de la semilla de los dos tipos de moringa inició a los 8 días después de la siembra, siendo la semilla proveniente de cápsula larga la que presentó el mayor porcentaje de germinación (80\%), mientras que en cápsula corta fue de $65 \%$ (Figura 1). La prueba T-Student (5.9325) indicó diferencias altamente significativas para las medias de esta variable $(p \leq 0.01)$ entre los tipos de moringa. En vivero las plántulas emergieron a los 18 días después de la siembra, el porcentaje de emergencia fue del $70 \%$ en cápsula larga y de $67.7 \%$ en cápsula corta (Tabla 1), sin diferencias significativas entre los dos tipos de moringa (0.776).

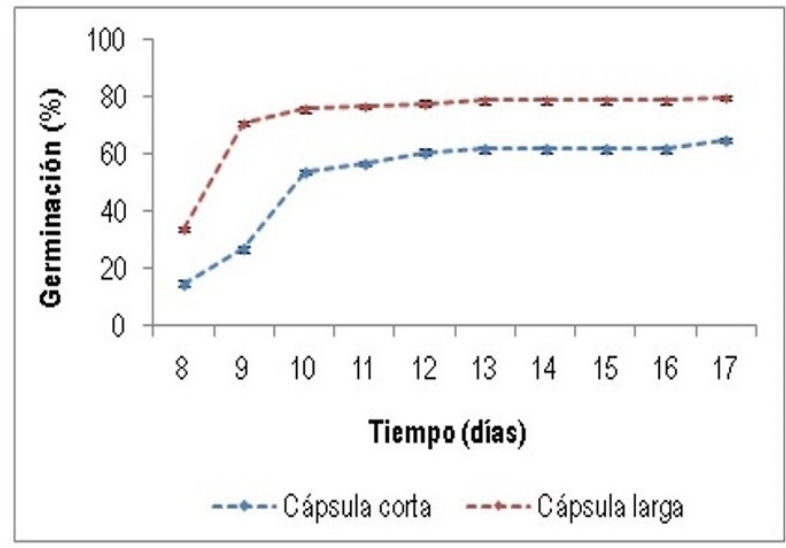

Figura 1. Porcentaje de germinación de semilla de dos tipos de cápsula de Moringa oleifera.

Tabla 1. Porcentaje de emergencia, índice de velocidad de emergencia (IVE) y velocidad de crecimiento (VC) de dos tipos de cápsula de Moringa oleifera.

\begin{tabular}{lccc}
\hline Tipo & $\begin{array}{c}\text { Emergencia } \\
(\%)\end{array}$ & $\begin{array}{c}\text { IVE } \\
\left(\text { plantas día }^{-1}\right)\end{array}$ & $\begin{array}{c}\text { VC } \\
\left(\mathrm{mm} \mathrm{dí}^{-1}\right)\end{array}$ \\
\hline Cápsula corta & 67.70 & $2.05 \pm^{*} 0.18$ & 1.24 \\
Cápsula larga & 70.00 & $2.01 \pm 0.10$ & 2.02 \\
\hline${ }^{*} \pm$ Desviación estándar. & &
\end{tabular}

El tipo de moringa de cápsula larga en las evaluaciones de altura de planta, tuvo un crecimiento mayor en comparación con la cápsula corta, con diferencias estadísticas $(p \leq 0.01)$. La altura observada en cápsula larga fue de $7.3,8.9$ y $10.2 \mathrm{~cm}$ y en la cápsula corta de $7.0,8.0$ y $8.8 \mathrm{~cm}$, presentando el mayor crecimiento las plantas provenientes de semilla de cápsula larga (Figura 2). En la Tabla 1 se presenta el índice de velocidad de emergencia (IVE) y velocidad de crecimiento (VC), donde se observan diferencias mínimas. Las plantas de moringa provenientes de cápsula corta tuvieron un IVE de $2.05 \pm 0.18$, mientras que en cápsula larga fue de $2.01 \pm 0.10$. La VC para cápsula larga fue de $2.02 \mathrm{~mm}$ día $^{-1}$ y para cápsula corta de $1.24 \mathrm{~mm}$ día $^{-1}$.

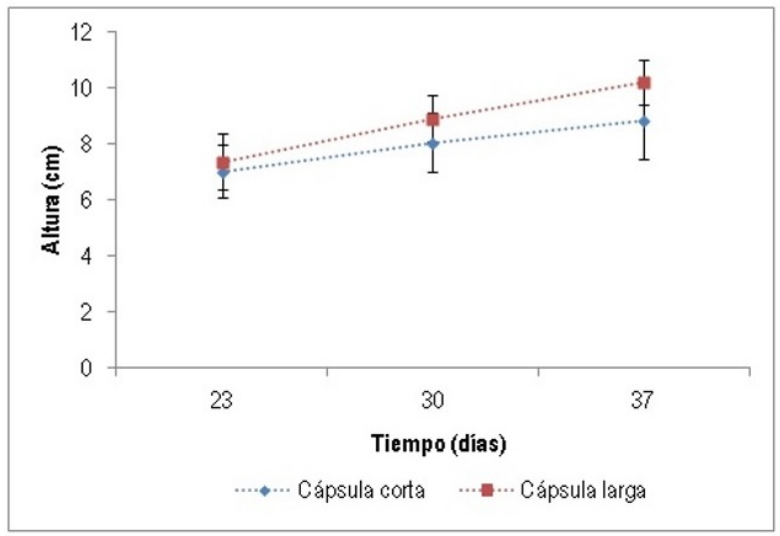

Figura 2. Altura de planta en vivero de dos tipos de cápsula de Moringa oleifera.

En el medio de crecimiento utilizado para el desarrollo de las plántulas, se identificaron nematodos del género Dorylaimus (Figura 3), con largo entre 3 y $5 \mathrm{~mm}$ de la cola al falso estilete biselado. También se encontraron nematodos del género Rhabditis, caracterizados por una cabeza con una cavidad bucal con tres protuberancias y un phasmidio que se encuentra cerca de la parte reproductora, el cual se utiliza en control biológico, debido que se alimenta de hongos y bacterias fitopatógenas. Dentro de los hongos se identificó al género Fusarium en el $70 \%$ de las semillas con testa, sin testa y en la testa sin semilla de moringa de cápsula corta, mientras que en cápsula larga solo se detectó en un 15\% (Figura 4). La especie Fusarium oxysporum Schlecht, se caracteriza por un crecimiento de micelio enmarañado aéreo, primero blanco y posteriormente de color morado. Se observaron microconidios cortos y ramificados, que 


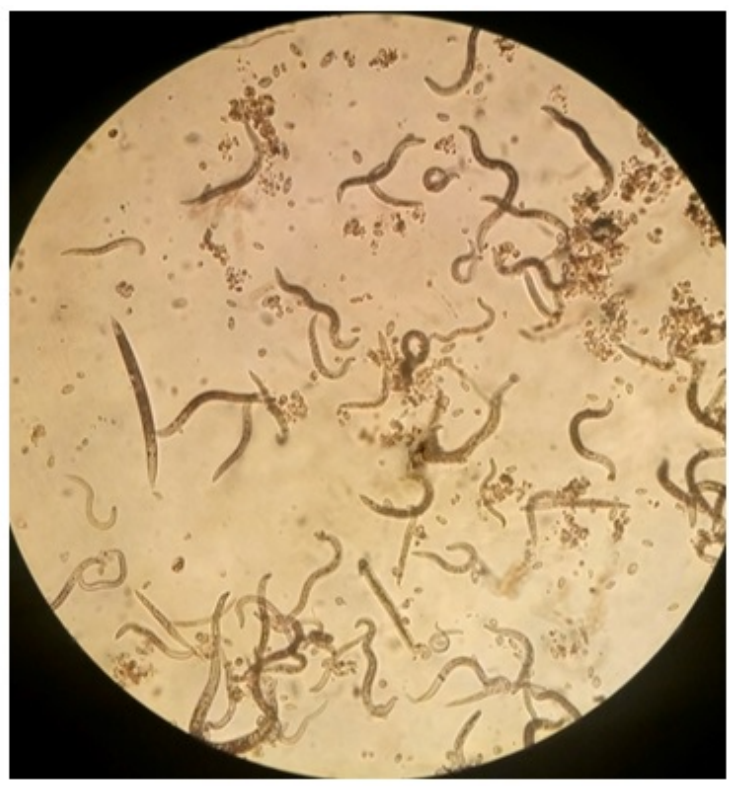

Figura 3. Nematodos Dorylaimus presente en peat most.
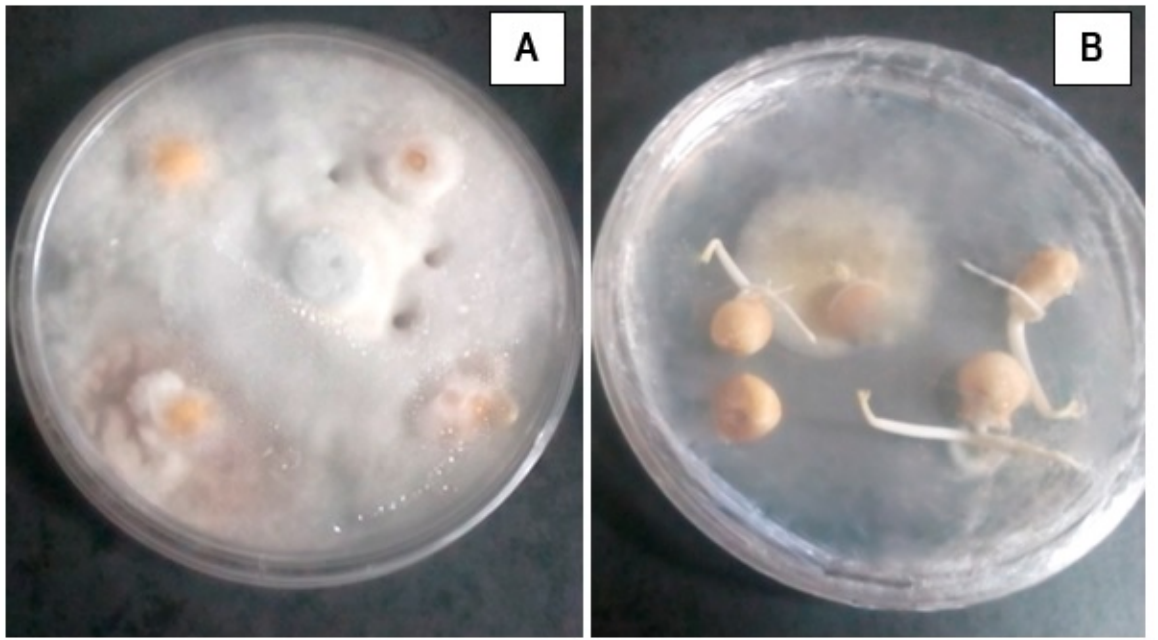

Figura 4. Fusarium oxysporum Schlecht en semilla de moringa. A) cápsula corta, B) cápsula larga.

son las características más importantes de F. oxysporum.

\section{Adaptación en condiciones de campo}

Las plantas de moringa no mostraron estrés durante el trasplante ni los días siguientes, mientras que las plantas de zacate buffel se marchitaron al ser trasplantadas, pero $5 \circ 6$ días después reanudaron el crecimiento. Los porcentajes de sobrevivencia al trasplante fueron del 97 y $100 \%$ para moringa y buffel, respectivamente (Tabla 2). Pero al final del verano debido a la competencia del zacate buffel, la sobrevivencia de la moringa osciló entre 1.03 y $5.12 \%$ en las repeticiones. Mientras que el zacate buffel creció de forma rápida con las temperaturas altas de final de primavera y el verano $\left(35^{\circ} \mathrm{C}\right)$, que mataron a la mayoría de las plantas de moringa. Todos los árboles que tuvieron competencia con zacate buffel fueron inca- 
Tabla 2. Porcentaje de establecimiento y sobrevivencia en tres densidades de población de Moringa oleifera en asociación con zacate buffel.

\begin{tabular}{|c|c|c|c|c|c|c|c|c|}
\hline \multirow[t]{2}{*}{ Especie } & \multirow{2}{*}{$\begin{array}{c}\text { Establecimiento } \\
(\%)\end{array}$} & \multirow{2}{*}{\multicolumn{3}{|c|}{$\begin{array}{c}\text { Sobrevivencia } \\
(\%)\end{array}$}} & \multirow{2}{*}{$\begin{array}{c}\text { Densidad } \\
\text { árboles parcela }^{-1}\end{array}$} & \multicolumn{2}{|c|}{ Competencia Intraespecífica } & \multirow{2}{*}{$\begin{array}{l}\text { Frutos } \\
\text { (No.) }\end{array}$} \\
\hline & & & & & & Sobrevivencia (\%) & Altura (m) & \\
\hline & & \multicolumn{2}{|c|}{ Competencia Interespecífica } & Invierno & & & & \\
\hline & & con buffel & sin buffel & & & & & \\
\hline Moringa & 97 & 5.29 & 65.39 & 32.27 & 15 & 31.11 & 3.15 & 1 \\
\hline \multirow[t]{2}{*}{ Zacate Buffel } & 100 & 100 & 100 & 100 & 40 & 30.83 & 4.22 & 48 \\
\hline & & & & & 140 & 14.04 & 3.03 & 36 \\
\hline
\end{tabular}

paces de sobrevivir, sobreviviendo solo los árboles que no tuvieron competencia con el zacate buffel en los surcos orilleros o cabeceras, mientras que el híbrido de zacate buffel mantuvo el $100 \%$ de su población original. Los árboles de moringa en la repetición sin competencia del zacate buffel crecieron de forma rápida al final de primavera y en el inicio de verano, superando en altura a las plantas de moringa con competencia de zacate buffel, con sobrevivencia promedio de las densidades de población de 15, 40 y 140 árboles por parcela del 31.11, 30.83 y $14.04 \%$, respectivamente. El análisis de varianza no detectó diferencias significativas entre las medias. Con sobrevivencia promedio de los árboles del 0.47, 10.11 y $65.39 \%$ en las tres repeticiones. La media de la repetición III fue mayor y estadísticamente diferente a las otras dos medias que fueron iguales, debido a que no tuvo competencia con el zacate buffel. Las temperaturas nocturnas frescas del otoño desaceleraron el crecimiento de los árboles de moringa hasta detenerlo de forma completa a fines de noviembre, pero debido a que pocos árboles produjeron flores, todo el crecimiento fue vegetativo. Para fines de diciembre, las plantas de moringa perdieron todo el follaje y entraron en latencia al igual que el zacate buffel.

La altura de los árboles de moringa indica competencia al interior de la especie, la densidad de población de 140 árboles por parcela tuvo el crecimiento promedio más bajo con 3.03 y $2.60 \mathrm{~m}$. De los 234 árboles, solamente 11 (4.7\%) crecieron $4.00 \mathrm{~m}$ o poco más, el árbol más alto midió 4.45 $\mathrm{m}$, de los 11 árboles nueve estuvieron en densidades de población de 140 y 40 árboles por parcela. Ningún árbol alcanzó los $4.00 \mathrm{~m}$ en las densidades de población de 15 y 140. La altura promedio de todos los árboles fue de $2.86 \mathrm{~m}$; midiendo dos árboles menos de $1.00 \mathrm{~m}$ (0.854\%), 20 entre 1.00 y $1.99 \mathrm{~m}$
(8.547\%), 103 entre 2.00 y 2.99 m (44.017\%), 98 entre 3.00 y $3.99 \mathrm{~m}(41.88 \%)$ y 11 entre 4.00 y 4.45 $\mathrm{m}(4.7 \%)$ (Figura 5). El valor de $X^{2}$ para la proporción 2/3:1/3 fue 0.097 con probabilidad de ocurrir por azar del $97.5 \%$, mientras que el análisis de $X^{2}$ aplicado a las subpoblaciones de 51 y 134 árboles por separado, los datos observados también se ajustaron a la proporción teórica 2/3:1/3 que se espera cuando una variable tiene una distribución normal. Todas las plantas de zacate buffel tuvieron una altura promedio de $1.00 \mathrm{~m}$, debido a que es una especie apomíctica obligada que se caracterizan por ser genéticamente uniformes e idénticas al progenitor femenino. La sobrevivencia al invierno de los árboles de moringa fue del $32.27 \%$, pero este porcentaje disminuyó a $31.44 \%$ a finales de verano del mismo año. Por el contrario, el $100 \%$ de las plantas de zacate buffel continuaron su desarrollo (Tabla 2).

Con respecto a la producción de frutos, de los 228 árboles de moringa vivos a principios del otoño de 2015, solo 22 produjeron una o más cápsulas, lo que corresponde al $9.6 \%$ del total de árboles. EI número de árboles con cápsula incrementó con el aumento de árboles en cada unidad experimental, pero el porcentaje promedio de árboles productivos fue de $9.33 \%$. En la densidad de población de 15 plantas, de 11 árboles solo uno produjo una cápsula; en la densidad de 140 plantas por parcela, de 53 árboles 5 de ellos produjeron 1, 4, 5, 8 y 10 cápsulas, en la densidad de 40 plantas por parcela con 34 árboles solo tres produjeron cápsulas con 1, 3 y 44 cápsulas que es la mayor cantidad observada. Mientras que en la densidad de población de 140 plantas por parcela con 130 árboles, 13 produjeron frutos, ocho con 1 cápsula, uno con 2 , dos con 6 , uno con 8 y uno con 13 cápsulas. 


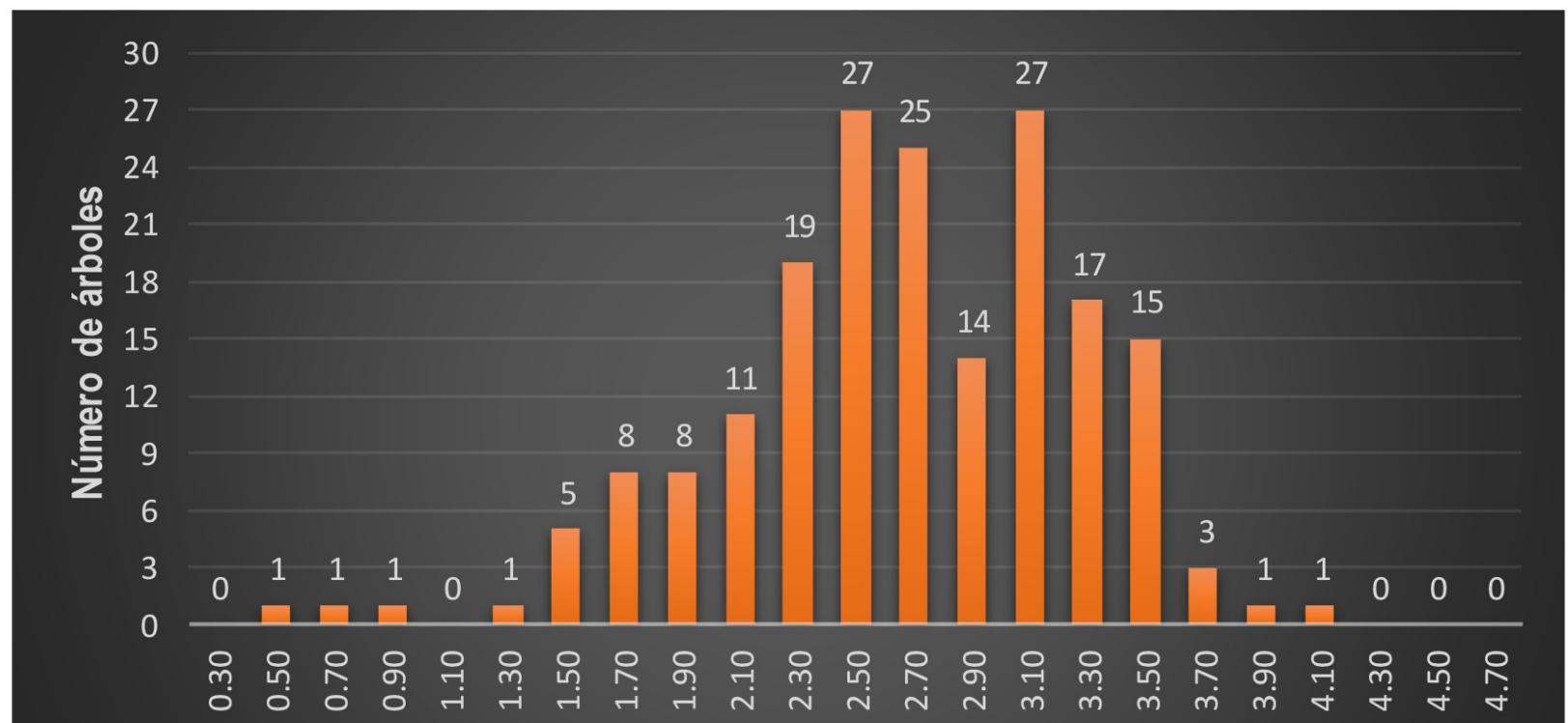

Altura de árboles de moringa (m)

Figura 5. Distribución normal de la altura de árboles de moringa.

\section{DISCUSIÓN}

\section{Laboratorio y vivero}

Se observó alta tasa de germinación en la semilla de moringa, $80 \%$ en cápsula larga y $65 \%$ en cápsula corta. Al respecto, Jahn et al. (1986) y, Nautiyal y Venhataraman (1987) reportan que la tasa de germinación varía de 60 a 90\% en moringa; mientras que Toral et al. (2013) reportan valores entre 49 y $84 \%$ para diferentes genotipos de moringa, y Pallavi et al. (2015) valores de $96 \%$ en semilla con testa y sin testa. Mientras que otros estudios reportan que la semilla almacenada por más de seis meses reduce su germinación (Fotouo et al. 2015). Pero los porcentajes de germinación de esta investigación son altos, considerando que se utilizó semilla de 1.5 años de almacenamiento a temperatura ambiente $y$ sin tratamiento previo. Por lo que factores genéticos, mecánicos, físicos, fisiológicos, tiempo y las condiciones de almacenamiento están involucrados en la germinación y emergencia de M. oleifera. Al respecto, algunos autores mencionan que un tratamiento pregerminativo a la semilla de moringa, promueve el rompimiento de la latencia, lo que incrementa la tasa de germinación (Padilla et al. 2012). Por el contrario, otros indican que no son necesarios los pretratamientos, ya que no incrementan el porcentaje de germinación (Candelaria-Martínez et al. 2019), e incluso algunos tratamientos disminuyen la germinación (Barraza 2017). La temperatura y el tipo de substrato son factores importantes que ayudan al proceso de germinación de la semilla (Medina et al. 2007, Pérez 2010). Siendo la temperatura fundamental para la germinación y establecimiento en campo (Bewley y Black 1994, Meza-Carranco et al. 2016), debido a que incrementa la velocidad de absorción del agua y por ende ocurren reacciones químicas que mejoran la germinación (Carvalho y Nakagawa 2012), emergencia y vigor de las plantas (Navarro et al. 2015).

Las plántulas de moringa emergieron a los 18 días después de la siembra, probablemente la temperatura de -2 a $-4{ }^{\circ} \mathrm{C}$ a finales del mes de marzo afectaron la emergencia, debido a que la planta es de clima cálido. Al respecto, Padilla et al. (2012), reportan el inicio de la emergencia entre 11 y 15 días después de la siembra, lo que difiere de lo reportado por otros autores (Medina et al. 2007, Toral et 
al. 2013). Sobre la germinación Nonagaki (2006), menciona que todas las semillas tienen sensores que les permiten detectar los cambios ambientales y las condiciones en que se encuentran determinan el éxito de la germinación, emergencia, y establecimiento. Los porcentajes de emergencia obtenidos para las plantas de cápsula larga y corta fueron del 70 y $67.7 \%$, respectivamente. Estos valores son altos tomando en cuenta las condiciones y tiempo de almacenamiento de la semilla. Por lo que los resultados obtenidos son superiores a los reportados por Barraza (2017) de $60,54,37$, y $28 \%$ en diferentes tratamientos, pero son similares a lo reportado por Candelaria-Martínez et al. (2019) de 80.0, 78.4, y $73.5 \%$. Los porcentajes de germinación entre los tipos de cápsula indica que el tamaño del fruto no influye en la emergencia de las plántulas, lo que coincide con Meza-Carranco et al. (2016) quienes no encontraron efecto del tipo de cápsula.

La moringa es una planta de rápido desarrollo vegetativo que expresa su mayor crecimiento 20 días después de la siembra (Toral et al. 2013). Al respecto, Medina et al. (2007) compararon el crecimiento de $M$. oleifera y Leucaena leucocephala en fase de vivero observando crecimientos de 53.20 y $45.00 \mathrm{~cm}$ de altura a las 13 semanas de siembra. Mientras que Toral et al. (2013) reportan alturas de planta de 17.50, 16.80 y $16.70 \mathrm{~cm}$ a los 40 días después de la siembra. Valores que son superiores a los obtenidos en el presente trabajo, lo que se puede deber a las bajas temperaturas que ocurrieron durante el desarrollo del experimento, las cuales afectaron el crecimiento.

Con respecto, al IVE se encontraron diferencias mínimas entre los dos tipos de moringa, valores que son bajos comparados con los reportados por Cardoso et al. (2006), quienes mencionan que el IVE se incrementa si la semilla se siembra a $2 \mathrm{~cm}$ de profundidad y se coloca con el ápice hacia arriba y de costado. Aun con las diferencias en la VC en las dos variantes de moringa (2.02 mm para cápsula larga y $1.24 \mathrm{~mm}$ para cápsula corta), estos valores son menores que $0.43,0.41,0.42$ y $0.31 \mathrm{~cm}$ día ${ }^{-1}$ a los 40 días después de la siembra (Toral et al. 2013). Mientras que Pérez et al. (2010) observaron valores similares con semilla sin escarificar, pero con semilla escarificada tuvieron valores de $0.57 \mathrm{~cm}$ día ${ }^{-1}$, creando de esta forma condiciones favorables para un incremento en el crecimiento de las plántulas. No se observó efecto del tamaño de cápsula en la germinación, altura de planta, y velocidad de crecimiento, por lo que las diferencias observadas en estas variables probablemente se deban al daño causado por $F$. oxysporum, ya que la semilla de vaina corta presentó un $70 \%$ de contaminación que le impidió un desarrollo normal. La presencia de F. oxysporum en la semilla de moringa posiblemente se deba al tipo y periodo de almacenamiento de la semilla, ya que los estudios relacionados con fitopatógenos en semilla de M. oleifera, reportan ataque de F. oxysporum, y la presencia de 47 especies microbianas de 26 géneros, siendo las más frecuentemente Phoma sp., Rhizopus stolonifer, Aspergillus fumigatus, Fusarium solani, A. niger, F. semitectum, Phomopsis sp y Chaetomium globusumun (Martínez et al. 2013). Sobre los nematodos del género Dorylaimus se sabe que atacan a plantas y animales, y se desarrollan en suelos húmedos (Mulvey y Anderson 1979). Debido al daño que producen, probablemente causaron la muerte y pérdida de vigor de las plantas en vivero, aunque se reporta que la planta de moringa son resistentes a daños por nematodos (Padilla et al. 2017).

\section{Adaptación en campo}

La alta sobrevivencia al trasplante de la moringa y el zacate buffel, se debe a que está técnica asegura un buen porcentaje de establecimiento de las plantas. Sin embargo, la diferencia entre ambas especies se puede explicar por su tipo de raíz, ya que en moringa es tuberosa (Olson 2010, Olson y Fahey 2011) y en el zacate buffel es fibroso (Robles et al. 1990), además de que la tolerancia a la sequía se atribuye a su sistema radicular profundo, que les permite a estas especies aprovechar de forma eficiente los nutrientes y la humedad del suelo (Skerman y Riveros 1990, Medina et al. 2007). Los resultados indican competencia interespecífica, debido a que en el establecimiento se encontró una mayor mortalidad de las plantas de moringa, en aquellas unidades experimentales donde se asoció con zacate buffel, ya que esta especie posee rizomas que le confieren 
mayor tolerancia a heladas y por lo tanto mayor sobrevivencia que otras especies (Bogdan 1997). Así mismo, las plantas de moringa presentaron competencia intraespecifica por nutrientes, agua y luz, lo que se manifestó en plantas de menor altura en aquellas parcelas donde la densidad de población fue mayor. La competencia por radiación solar entre plantas de moringa ha sido reportada por Reyes et al. (2006). Mientras que Meza-Carranco et al. (2016) encontraron asociación negativa entre la densidad de población y la altura de planta de moringa, además de que mayor densidad de población también conduce a mayor porcentaje de mortalidad.

\section{CONCLUSIONES}

El tipo de cápsula larga presentó mayor porcentaje de germinación e índice de velocidad de crecimiento, que el tipo cápsula corta. Sin importar el tipo de cápsula la semilla fue atacada por $F$. oxysporum aunque la presencia fue mayor en semilla proveniente de cápsula corta. En vivero se identificó la presencia de nematodos del género Dorylaimus en el sustrato, los cuales pudieron causar el marchitamiento de las plantas. La evaluación en campo indica que Moringa oleifera tiene potencial de adaptación en la región semiárida del norte de México, donde podría servir para minimizar los efectos del cambio climático; pero se requiere determinar la densidad de población óptima, ya que se observó competencia intra e interespecífica.

\section{AGRADECIMIENTOS}

Al CONACYT por la beca otorgada para realizar estudios de postgrado. Este proyecto fue apoyado financieramente por la Secretaría de Agricultura, Pesca y Ganadería a través del Proyecto: FON.SEC. SAGARPA-CONACYT CV2015-4-266936. Además de apoyo financiero parcial de la Universidad Autónoma de Coahuila y la Universidad Autónoma Agraria Antonio Narro.

\section{LITERATURA CITADA}

Barraza AF (2017) Germinación de semillas de moringa (Moringa oleifera Lam.) en diferentes tiempos de imbibición en agua. Revista UDCA Actualidad \& Divulgación Científica 20: 71-77.

Bewley DD, Black M (1994) Seeds: physiology of development and germination. Plenum. New York. 467p.

Bogdan AV (1997) Pastos tropicales y plantas de forraje. Ed AGT editor. México. 461p.

Candelaria-Martínez B, Vera-López J, Chiquini-Medina RA, Flota-Bañuelos C (2019) Germination responses of Moringa oleifera seeds treated with two scarification methods. Revista Bio Ciencias 6 e407. Doi: 10.15741/revbio.06.e407.

Cardoso RM, Daniel CD, Carvalho MV, Sousa AH (2006) Profundidad y posición de la semilla en la emergencia y desarrollo de plántulas de moringa. Centro Agrícola 33: 5-8.

Carvalho NM, Nakagawa J (2012) Sementes: ciencia, tecnología e producao. 5ta Ed. FUNEP: Jaboticabal 590p.

Castillo-López RI, León-Félix J, Angulo-Escalante MA, Gutiérrez-Dorado R, Muy-Rangel MD, Basilio-Herrera $\mathrm{J}(2017)$ Nutritional and phenolic characterization of Moringa oleifera leaves grown in Sinaloa, Mexico. Pakistan Journal of Botany 49: 161-168.

Cepeda SM (1996) Nematología Agrícola. Editorial Trillas. México. 319p.

CONAGUA (2015) Servicio Meteorológico Nacional. México. https://smn.conagua.gob.mx/es/pronostico-deltiempo-por-municipios. Fecha de consulta: 17 de febrero de 2020. 
Conant RT, Paustian K, Del Grosso SJ, Parton WJ (2005) Nitrogen pools and fluxes in grassland soils sequestering carbon. Nutrient Cycling in Agroecosystems 71: 239-248.

Easterling WE, Aggarwal PK, Batima P, Brander KM, Erda L, Howden SM, Howden, Kirilenko A, Morton J, Soussana J-F, Schmidhuber J, Tubiello FN (2007) Food, fibre and forest products. In: Parry ML, Canziani OP, Palutikof JP, Van der Linden PJ, Hanson CE (Eds.) Climate change: Impacts, adaptation and vulnerability. Contribution of Working Group II to the Fourth Assessment Report of the Intergovernmental Panel on Climate Change, Cambridge University Press. Cambridge, UK. pp: 273-313.

Espinoza-Ahumada CA, Gallegos-Morales G, Hernández-Castillo FD, Ochos-Fuentes YM, Cepeda-Siller M, Castillo-Reyes F (2019) Antagonistas microbianos a Fusarium spp., como agente causal de pudrición de raíces y tallos en melón. Ecosistemas y Recursos Agropecuarios 6: 45-55.

Fotouo MH, Toit ES, Robbertse PJ (2015) Germination and ultrastructural studies of seeds produced by a fastgrowing, drought-resistant tree: implications for its domestication and seed storage. AoB PLANTS 7: 1-12.

Gómez de la FE, Díaz SH, Saldívar FA, Briones EF, Vargas TV, Grant WE (2007) Patrón de crecimiento de pasto buffel [Pennisetum ciliare L. (Link.) Sin. Cenchrus ciliaris L.] en Tamaulipas, México. Técnica Pecuaria en México 45: 1-17.

Gómez-Martínez M, Ascacio-Valdés JA, Flores-Gallegos AC, González-Domínguez J, Gómez-Martínez S, Aguilar CN, Morlett-Chávez JA, Rodríguez-Herrera R (2020) Location and tissue effects on phytochemical composition and in vitro antioxidant activity of Moringa oleifera. Industrial Crops \& Products 151: 1-8.

Gutiérrez M, Ordoñez A, Muñoz A, Galicia A, Hernández T, Jiménez I (2013) Importancia de las emisiones de gases efecto invernadero del sector agricultura en México. Revista VITA 6: 26-27.

Hernández LG, Aparicio JR, Mancini F (2018) Pobreza y derechos sociales en México. Consejo Nacional de Evaluación de la Política de Desarrollo Social. UNAM. 735p.

Jahn S, Musnad HA, Burgstaller H (1986) The tree that purifies water: Cultivating multipurpose Moringaceae in the Sudan. Unasylva 38: 23.

Krishnamurthy YL, Shashikala J, Shankar NB (2008) Antifungal potential of some natural products against Aspergillus flavus in soybean seeds during storage. Journal of Stored Products Research 44: 305-309.

Lakshmipriya G, Doriya K, Santos KD (2016) Moringa oleifera: A review on nutritive importance and its medicinal application. Food Science and Human Wellness 5: 49-56.

Magaña VO, Neri C (2012) Cambio climático y sequías en México. Ciencia 63: 26-35.

Maguire JD (1962) Speed of germination-aid in selection and evaluation for seedling emergence and vigor. Crop Science 2: 176-177.

Martínez PE, Cantillo PT, García RD (2013) Microbiota asociada a lotes importados de semillas de moringa (Moringa oleifera). Fitosanidad 17: 125-129.

Medina M, García D, Clavero T, Iglesias J (2007) Estudio comparativo de Moringa oleifera y Leucaena leucocephala durante la germinación y la etapa inicial de crecimiento. Zootecnia Tropical 25: 83-93.

Mendieta-Araica B, Sporndly E, Reyes-Sánchez N, Salmerón-Miranda F, Halling M (2013) Biomass production and chemical composition of Moringa oleifera under different planting densities and level of nitrogen fertilization. Agroforestry Systems 87: 81-91.

Meza-Carranco Z, Olivares-Sáenz E, Gutiérrez-Ornelas E, Bernal-Barragán H, Aranda-Ruiz J, Vázquez-Alvarado RE, Carranza-de la Rosa R (2016) Crecimiento y producción de biomasa de moringa (Moringa oleifera Lam.) bajo las condiciones climáticas del Noreste de México. Tecnociencia Chihuahua 10: 143-153. 
Mulvey RH, Anderson RV (1979) Benthic species of Dorylaimus Dujardin, 1845 (nematode: Dorylaimidae) and Arctidorylaimus n. gen. from the Mackenzietories. Canada. Canadian Journal of Zoology 57: 745-755.

Navarro M, Cicero SM, Gomes JFG (2015) Determination of the germination temperature of Moringa oleifera seeds with support of vigor tests. Cuban Journal of Agricultural Science 49: 509-514.

Nautiyal BP, Venhataraman KG (1987) Moringa (drumstick) - an ideal tree for social forestry: growing conditions and uses - Part I. Myforest 23: 53-58.

Nonagaki H (2006) Seed germination-The biochemical and molecular mechanisms. Breeding Science 56: 93105.

Olson ME (2010) Moringaceae: drumstick family. In: Flora of North America North of Mexico. Flora of North America Editorial Committee, eds. New York, New York and Oxford. 7: 167-169.

Olson ME, Alvarado LO (2016) ¿Dónde cultivar el árbol milagro, Moringa oleifera, en México? Un análisis de su distribución potencial. Revista Mexicana de Biodiversidad 87: 1089-1102.

Olson ME, Fahey J (2011) Moringa oleifera: un árbol multiusos para las zonas tropicales secas. Revista Mexicana de Biodiversidad 82: 1071-1082.

Oswald A, Ransom JK (2002) Response of maize varieties to transplanting in striga-infested fields. Weed Science 50: 392-396.

Padilla C, Fraga N, Suárez M (2012) Efecto del tiempo de remojo de las semillas de Moringa (Moringa oleifera) en el comportamiento de la germinación y en indicadores del crecimiento de la planta. Revista Cubana de Ciencia Agrícola 46: 419-421.

Padilla C, Valenciaga N, Crespo G, González D, Rodríguez I (2017) Requerimientos agronómicos de Moringa oleifera (Lam.) en sistemas ganaderos. Livestock Research for Rural Development 29. Article 218.

Pallavi HM, Madalageri MB, Vishwanath K, Biradar IB Thattimani M (2015) Decortication to enhance seed value and standardization of quick viability test in drumstick (Moringa oleifera L.). Environment and Ecology 33: 1558-1561.

Pérez A, Sánchez T, Armengol N, Reyes F (2010) Características y potencialidades de Moringa oleifera, Lamarck. Una alternativa para la alimentación animal. Pastos y Forrajes 33: 1-10.

Pérez AR (2010) Moringa oleifera, una alternativa forrajera para Sinaloa. Fundación Produce Sinaloa A.C. SAGARPA. ResearchGate 19p.

Reyes NS, Ledin S, Ledin I (2006) Biomass production and chemical composition of Moringa oleifera under different management regimes in Nicaragua. Agroforestry Systems 66: 231-242.

Robles SRO, Eichelmann B, Alvarado OA (1990) Cultivo del zacate buffel (Cenchrus ciliaris L.). En: Robles SR (ed) Producción de granos y forrajes. Quinta edición. Ed. Limusa. México. pp: 442-445.

Ruiz CJA (2012) Adaptar la agricultura al cambio climático. En: Cambio climático. Revista Ciencia 63: 76-83.

Skerman PJ, Riveros F (1990) Tropical grasses. FAO Plant production and protection paper No. 23. First Ed. Food and Agriculture Organization of the United Nations. Rome. 832p.

Tesfay SZ, Modi AT, Mohammed F (2016) The effect of temperature in Moringa seed phytochemical compounds and carbohydrate mobilization. South African Journal of Botany 102: 190-196.

Toral O, Cerezo Y, Reino J, Santana H (2013) Caracterización morfológica de ocho procedencias de Moringa oleifera (Lam) en condiciones de vivero. Pastos y Forrajes 36: 409-416. 
Ecosistemas

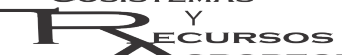

Recursos
Moringa: Comportamiento en invernadero y campo

Ecosist. Recur. Agropec. 7(2): e2408, 2020 https://doi.org/10.19136/era.a7n2.2408

Warham EJ, Butler LD, Sutton RC (1997) Ensayos para la semilla de maíz y de trigo: Manual de laboratorio. México. 84p. 